\title{
Comparative anatomical analysis of the transcallosal-transchoroidal and transcallosal-transforniceal-transchoroidal approaches to the third ventricle
}

\author{
João Luiz Vitorino Araujo, MD, PhD, ${ }^{1,2}$ José C. E. Veiga, MD, PhD, ${ }^{2}$ Hung Tzu Wen, MD, PhD, ${ }^{1}$ \\ Almir F. de Andrade, MD, PhD, ${ }^{1}$ Manoel J. Teixeira, MD, PhD, ${ }^{1}$ José P. Otoch, MD, PhD, ${ }^{1}$ \\ Albert L. Rhoton Jr., MD, ${ }^{3}$ Mark C. Preul, MD, ${ }^{4}$ Robert F. Spetzler, MD, ${ }^{4}$ and \\ Eberval G. Figueiredo, MD, PhD'
}

'Division of Neurosurgery, University of São Paulo Medical School; ²Discipline of Neurosurgery, Santa Casa de São Paulo Medical School, São Paulo, Brazil; 32Department of Neurological Surgery, University of Florida, Gainesville, Florida; and ${ }^{2}$ Division of Neurological Surgery, Barrow Neurological Institute, St. Joseph's Hospital and Medical Center, Phoenix, Arizona

OBJECTIVE Access to the third ventricle is a veritable challenge to neurosurgeons. In this context, anatomical and morphometric studies are useful for establishing the limitations and advantages of a particular surgical approach. The transchoroidal approach is versatile and provides adequate exposure of the middle and posterior regions of the third ventricle. However, the fornix column limits the exposure of the anterior region of the third ventricle. There is evidence that the unilateral section of the fornix column has little effect on cognitive function. This study compared the anatomical exposure afforded by the transforniceal-transchoroidal approach with that of the transchoroidal approach. In addition, a morphometric evaluation of structures that are relevant to and common in the 2 approaches was performed.

METHODS The anatomical exposure provided by the transcallosal-transchoroidal and transcallosal-transfornicealtranschoroidal approaches was compared in 8 fresh cadavers, using a neuronavigation system. The working area, microsurgical exposure area, and angular exposure on the longitudinal and transversal planes of 2 anatomical targets (tuber cinereum and cerebral aqueduct) were compared. Additionally, the thickness of the right frontal lobe parenchyma, thickness of the corpus callosum trunk, and longitudinal diameter of the interventricular foramen were measured. The values obtained were submitted to statistical analysis using the Wilcoxon test.

RESULTS In the quantitative evaluation, compared with the transchoroidal approach, the transforniceal-transchoroidal approach provided a greater mean working area (transforniceal-transchoroidal $150 \pm 11 \mathrm{~mm}^{2}$; transchoroidal $121 \pm 8$ $\mathrm{mm}^{2} ; \mathrm{p}<0.05$ ), larger mean microsurgical exposure area (transforniceal-transchoroidal $101 \pm 9 \mathrm{~mm}^{2}$; transchoroidal 80 $\pm 5 \mathrm{~mm}^{2} ; \mathrm{p}<0.05$ ), larger mean angular exposure area on the longitudinal plane for the tuber cinereum (transfornicealtranschoroidal $71^{\circ} \pm 7^{\circ}$; transchoroidal $64^{\circ} \pm 6^{\circ} ; p<0.05$ ), and larger mean angular exposure area on the longitudinal plane for the cerebral aqueduct (transforniceal-transchoroidal $62^{\circ} \pm 6^{\circ}$; transchoroidal $55^{\circ} \pm 5^{\circ} ; p<0.05$ ). No differences were observed in angular exposure along the transverse axis for either anatomical target (tuber cinereum and cerebral aqueduct; $p>0.05$ ). The mean thickness of the right frontal lobe parenchyma was $35 \pm 3 \mathrm{~mm}$, the mean thickness of the corpus callosum trunk was $10 \pm 1 \mathrm{~mm}$, and the mean longitudinal diameter of the interventricular foramen was $4.6 \pm 0.4 \mathrm{~mm}$. In the qualitative assessment, it was noted that the transforniceal-transchoroidal approach led to greater exposure of the third ventricle anterior region structures. There was no difference between approaches in the exposure of the structures of the middle and posterior region.

CONCLUSIONS The transforniceal-transchoroidal approach provides greater surgical exposure of the third ventricle anterior region than that offered by the transchoroidal approach. In the population studied, morphometric analysis established mean values for anatomical structures common to both approaches.

https://thejns.org/doi/abs/10.3171/2016.8.JNS16403

KEY WORDS neuroanatomy; comparative anatomy; neurosurgical procedure; fornix; third ventricle; corpus callosum; diagnostic technique 
$\mathrm{T}$ HE third cerebral ventricle is a narrow cavity that is centrally and deeply located. The structures surrounding the third ventricle represent less than $1 \%$ of the brain area. However, they are vital areas, because they participate in the endocrine, autonomic, and limbic systems. There are 3 main routes to access this region: superiorly, through the cortex and corpus callosum; ventrally, through the lamina terminalis; or dorsally, by opening the velum interpositum of the suprapineal recess. Manipulation of neural structures is therefore inevitable when accessing the third ventricle..$^{17,20,35,38,39}$

The transcallosal-transchoroidal approach to the third ventricle allows for adequate exposure of the middle and posterior regions of the ventricle. However, the fornix column limits exposure of the anterior region of the third ventricle. ${ }^{30,38}$

There is evidence that a unilateral lesion of the fornix column has little or no impact on memory. $2,4,8,15,22,25,28,30,43$ Various regions and structures participate in the memory circuit, and the fornix is just one of these structures. Changes to other parts of this circuit would therefore be necessary to establish a permanent memory deficit. ${ }^{16,18}$ In addition, there is evidence of good functional recovery in patients with bilateral lesions of the fornix; this recovery is associated with brain neuroplasticity and the activation of specific brain areas. ${ }^{26}$

To date, there have been no objective criteria for the choice of surgical approach to the third ventricle. This decision is fundamentally based on the neurosurgeon's experience, knowledge, and preferences. The proper choice of surgical approach results in lower morbidity and greater surgical safety. In this context, comparative anatomical studies that allow us to objectively recognize the limitations and advantages of a particular surgical approach are important for the selection and systematization of the surgical techniques used.

There is no evidence in the literature showing that unilateral section of the fornix column increases exposure of the brain structures. There is a possibility that this measure would not result in increased exposure. However, any significant increase in anatomical exposure due to unilateral sectioning of the fornix column could justify its systematic use as a viable alternative to accessing lesions located in the anterior region of the third cerebral ventricle.

The primary objective of the present study is to comparatively evaluate the surgical exposure afforded by the transcallosal-transchoroidal and transcallosal-transforniceal-transchoroidal approaches by measuring the surgical exposure area, working area, and angular exposure area. It also aims to qualitatively analyze the 2 approaches using microsurgical viewing of the exposed third ventricle floor structures. Second, a morphometric study of the relevant anatomical structures that are common to both techniques is performed.

\section{Methods}

The transcallosal-transchoroidal and transcallosaltransforniceal-transchoroidal approach methods were studied in 8 adult fresh cadavers without evidence of pathological brain changes, with an interval of less than 12 hours from death. The cadavers were obtained from the Death Verification System of the State of São Paulo, Brazil. Anatomical dissection was performed at the Microsurgery Laboratory, School of Medicine, University of São Paulo. Approval was obtained from the Ethics Committee of the University of São Paulo Medical School. The cadavers were placed supine on the operating table with the head fixed rigidly in a Sugita head frame (Mizuho) with a cervical flexion of $30^{\circ}$, simulating the surgical position. This rigid fixation prevented changes in head position.

Removal of the bone flap was systematically performed on the right side using high-speed drills and a craniotome (DePuy Synthes). Microsurgical procedures were performed with the use of a surgical microscope (Carl Zeiss), with magnifications ranging from $\times 6$ to $\times 40$, and appropriate neurosurgical instruments (Aesculap; Macom). Retraction of the medial aspect of the right frontal lobe was systematically performed using a static retraction system (Budde Halo, Ohio Medical Instrument). Measurements were obtained by a computerized neuronavigation system with a digital probe that had 4 spatial markers (Artis).

Dissection of 3 fixed brains was performed at the Department of Morphology, Santa Casa de São Paulo Medical School, with the purpose of illustrating the present study.

\section{Transcallosal-Transchoroidal Approach}

Three trepanations were performed in the right frontal region, simulating a surgical approach to the nondominant hemisphere. After ample exposure of the skull by transverse bicoronal incision to view the frontal, temporal, and parietal regions bilaterally, the surgical neuronavigation system (Artis) provided the measurement and standardization of trepanation sites. The first hole was drilled 5 $\mathrm{cm}$ in front of the coronal suture and $1 \mathrm{~cm}$ lateral to the sagittal suture, the second hole was drilled $2 \mathrm{~cm}$ posterior and $1 \mathrm{~cm}$ lateral to the sagittal suture, and the third hole was made at a point equidistant to the first 2 , in order to perform a craniotomy in the form of an isosceles triangle containing sagittal and coronal suture segments. With the aid of a high-speed craniotome, a bone flap of approximately $7 \times 6 \times 6 \mathrm{~cm}$ was obtained, exposing the superior sagittal sinus and dura mater. ${ }^{34}$

Dural opening was performed in an arc, because the pedicle was limited by the superior sagittal sinus. The dura mater was folded against the sinus and repaired with 4-0 PROLENE thread (Ethicon, Inc.) in the cerebral falx for medial retraction and to obtain additional space for exposure of the interhemispheric fissure.

The interhemispheric fissure was dissected using a microsurgical technique, with exposure of the corpus callosum and the 2 pericallosal arteries. Classic longitudinal callosotomy was performed between the 2 pericallosal arteries, with 2 -cm extension in the corpus callosum trunk region, guided by the neuronavigation system and using the contralateral coronal suture as a reference point. ${ }^{41}$ The callosotomy was performed $2 \mathrm{~cm}$ posterior to the coronal suture plane to maximize exposure of the third ventricle structures. The retractor was positioned on the right to allow lateral displacement of the right frontal lobe and corpus callosum, with a distance between the cerebral falx 


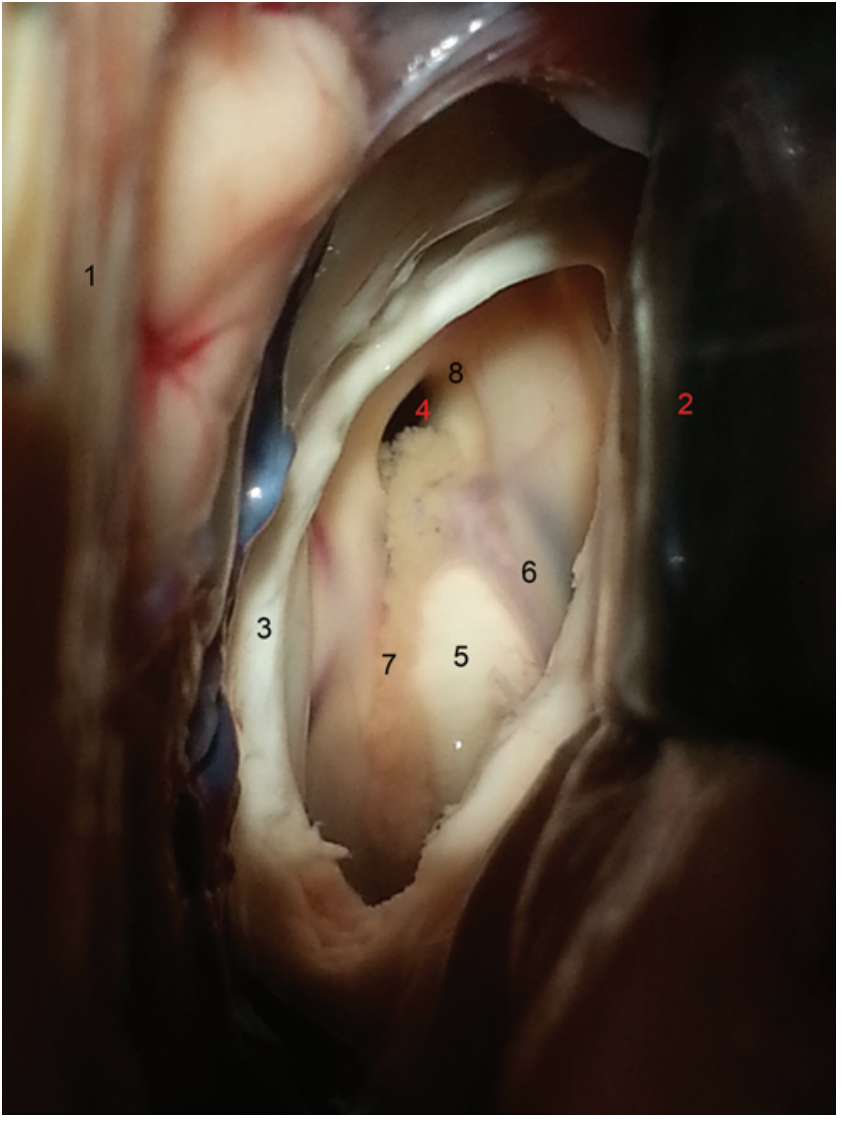

FIG. 1. Anatomical dissection of the specimen-superior microsurgical view of the right lateral ventricle in the transcallosal approach. $1=$ cerebral falx; 2 = retractor spatula withdrawing the right frontal lobe; $3=$ corpus callosum trunk; $4=$ interventricular foramen; $5=$ thalamus; $6=$ thalamostriate vein; $7=$ choroid plexus; $8=$ fornix column. Figure is available in color online only.

and spatula of $1.5 \mathrm{~cm}^{2}$. Anatomical right lateral ventricle structures relevant to the transchoroidal approach were then systematically identified as follows: the interventricular foramen, fornix column, choroid plexus, thalamus, and thalamostriate vein (Fig. 1).

Choroidal cleft dissection was performed by the tenia of the fornix, from the interventricular foramen to the choroid glomus, with a length of approximately $1.5-2.0 \mathrm{~cm} .{ }^{6}$ Next, the upper membrane of the choroidal screen was opened, with exposure of the internal cerebral veins and posterior-medial choroidal arteries. The lower choroidal screen membrane between the internal cerebral veins was then opened, exposing the choroid plexus of the third ventricle ceiling. Dissection continued, with exposure of the third ventricle cavity. In 3 selected cases, anterior septal vein sectioning was performed to increase the exposure of the third ventricle structures.

After the transchoroidal approach was complete, another retractor was strategically positioned to the left, allowing the retraction of $0.5 \mathrm{~cm}$ of the falx and the corpus callosum, establishing a corridor of $2 \mathrm{~cm}$ on the transverse plane, as determined by the neuronavigation system (Fig. 2). In specimens in which interthalamic adhesion obstructed proper viewing of the floor structures, a longitu-

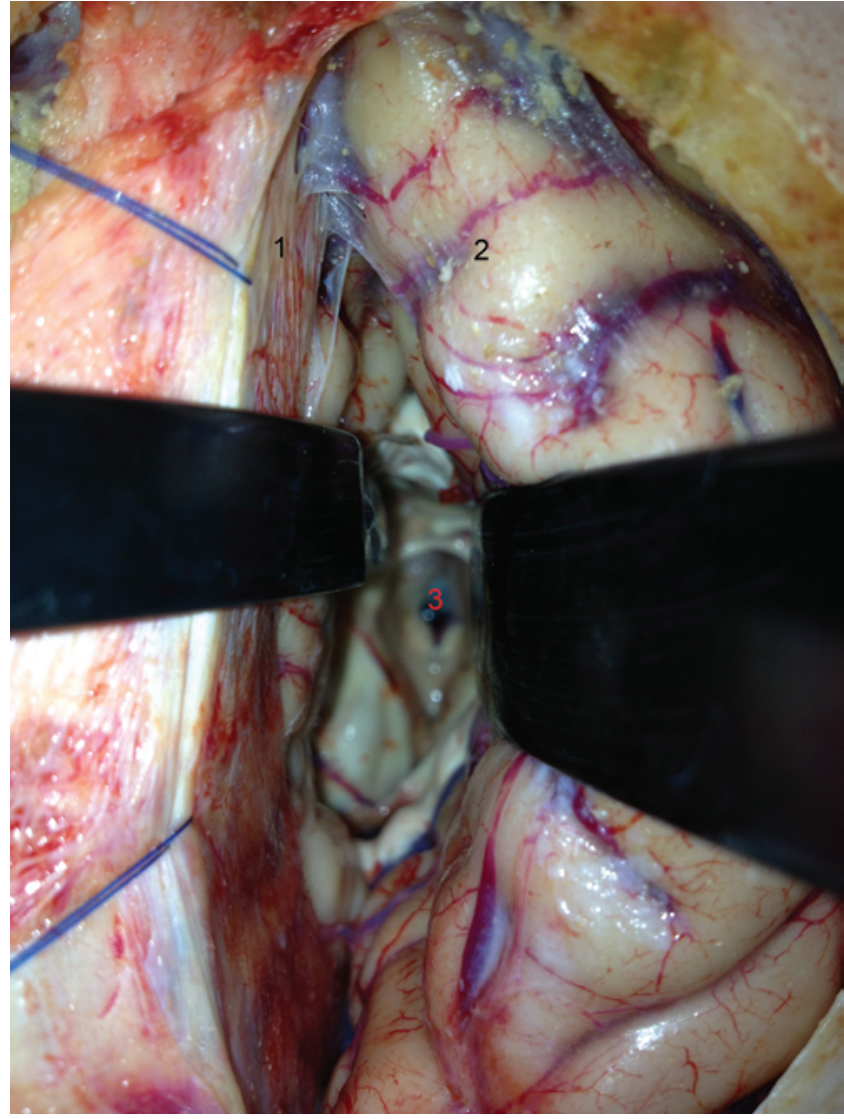

FIG. 2. Anatomical dissection of the specimen-superior macroscopic view after the transchoroidal approach showing the surgical corridor obtained after retractor placement. 1 = cerebral falx; 2 = right frontal lobe; 3 = anterior region of the third ventricle floor. Figure is available in color online only.

dinal section was performed using a No. 11 scalpel blade. ${ }^{6}$ Measurements were then taken using the neuronavigation system (Eximius, Artis).

\section{Transcallosal-Transforniceal-Transchoroidal Approach}

In the same specimen, unilateral sectioning of the fornix column was performed in its upper portion, near the angle formed between the body and the column. This technique is known as the transforniceal-transchoroidal approach. This method facilitates the proper evaluation of the 2 approaches without having to change the retractor position. Measurements were then taken using the neuronavigation system.

\section{Measurement Procedures}

Values were obtained using a computerized neuronavigation system with a digital probe that had 4 spatial markers. The head was fixed in a surgical head frame to ensure spatial stability in the same Cartesian system coordinates (Eximius System, Artis). The computer connected to the system stored data as $\mathrm{x}, \mathrm{y}$, and $\mathrm{z}$ coordinates of each previously defined point. The surgical retractors remained static while locating the target anatomical points, preventing measurement errors. The data acquired by the digital 
probe when touching the anatomical targets of interest were recorded by the system cameras. The neuronavigation system used has an intrinsic measurement error of 1 $\mathrm{mm}$. In the present study, we did not consider this value in the statistical analysis, given that this error is typically present in any neuronavigation system that uses a manual probe - that is, the error is inherent to the method used.

\section{Transcallosal-Transchoroidal Morphometry: Thickness of the Right Frontal Lobe Parenchyma and Corpus Callosum Trunk}

The following structures were evaluated by morphometry: thickness of the right frontal lobe parenchyma, thickness of the corpus callosum trunk, and diameter of the interventricular foramen.

To measure the thickness of the frontal lobe parenchyma, we used the following as references: the point on the cortical surface in the contralateral coronal suture plane and the sulcus of the corpus callosum. The corpus callosum trunk was measured in this same plane, using its upper and lower limits as references.

\section{Diameter of the Interventricular Foramen}

Due to the oval shape of the interventricular foramen, the diameter was measured along its longest axis, the longitudinal axis. The measured distance corresponds to the distance from the fornix column to the anterior pole of the thalamus.

\section{Comparative Analysis of the Transcallosal-Transchoroidal and Transcallosal-Transforniceal-Transchoroidal Approaches}

\section{Working Area and Surgical Exposure Area}

The working area was defined as the hexagonal area on the roof of the third ventricle obtained after dissection of the choroidal fissure and medial retraction of the ipsilateral fornix body in the transchoroidal approach. The defined points were as follows: ipsilateral fornix column (A); anterior tubercle of the thalamus (B); most posterior thalamic limit of the choroidal fissure (C); most distal point of the choroidal fissure, limited by the glomus of the choroid plexus (D); most posterior limit of the fornix body (E); and region of the fornix column adjacent to the venous angle (F) (Fig. 3). In the transforniceal-transchoroidal approach, Point A was the most anterior limit obtained after unilateral section of the fornix column, and the other points were the same as in the transchoroidal approach.

The surgical exposure area was defined as the hexagonal area on the third ventricle floor limited by the following points: the most anterior point exposed (1); point immediately most lateral and anterior to the floor of the right third ventricle (2); point immediately most lateral and posterior to the floor of the right third ventricle (3); cerebral aqueduct (4); point immediately most lateral and posterior to the floor of the left third ventricle (5); and point immediately most lateral and anterior to the floor of the left third ventricle (6) (Fig. 4). In all dissections, we were able to see the cerebral aqueduct, which corresponds to the most posterior structure of the third ventricle floor.

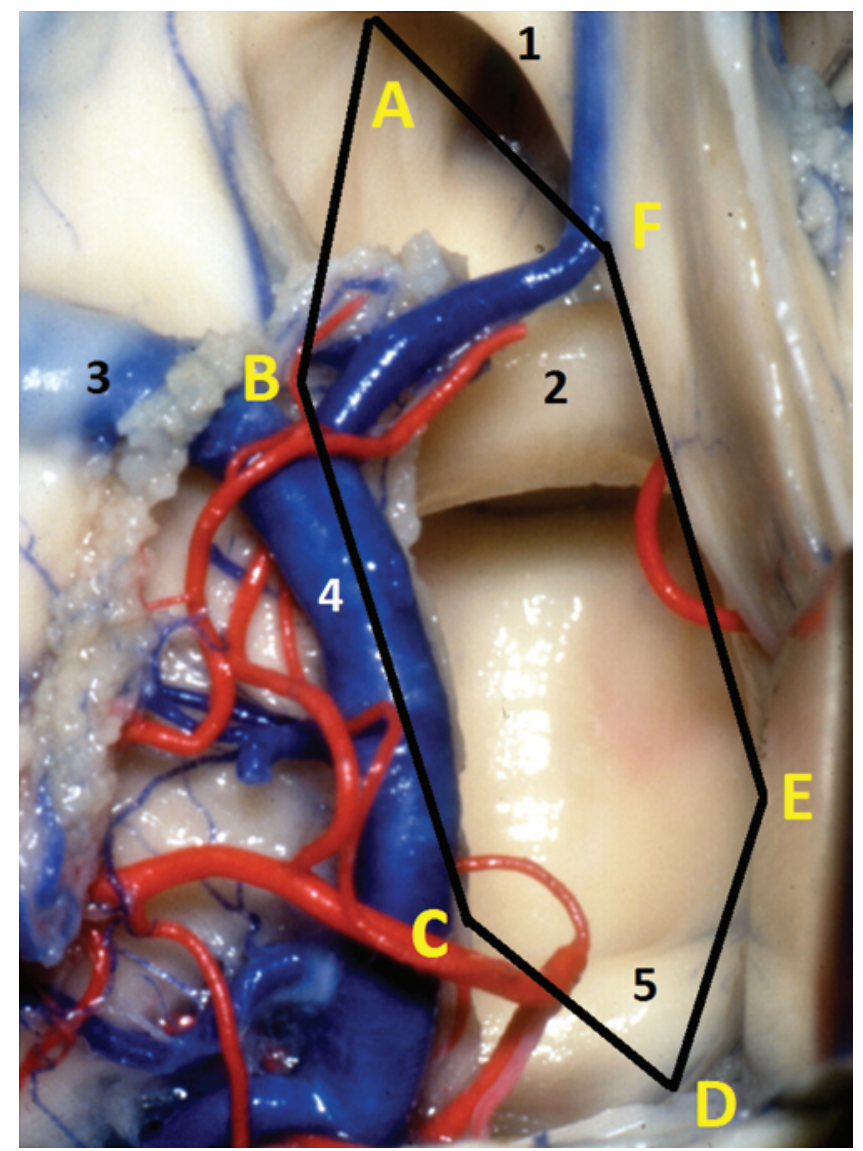

FIG. 3. Schematic representation of the working area in the transchoroidal approach in brain fixed in formaldehyde. The 6 points corresponding to anatomical points of interest and delimiting a hexagonal area are as follows: ipsilateral fornix column (A), anterior tubercle of the thalamus (B), most posterior thalamic limit of the choroidal fissure (C), most distal point of the choroidal fissure, limited by the glomus of the choroid plexus (D), most posterior limit of the fornix body $(E)$, and region of the fornix column adjacent to the venous angle $(F)$. $1=$ anterior septal vein; $2=$ interthalamic adherence; $3=$ thalamostriate vein, $4=$ internal cerebral vein; 5 = posterior commissure. Adapted from Sekhar LN, Fessler RG: Atlas of Neurosurgical Techniques: Brain. New York: Thieme, 2006. Copyright Thieme. Published with permission. Figure is available in color online only.

Thus, we used this structure as a fixed and common point in the 2 surgical approaches. The selection of these points defined a hexagonal area that reproduces microsurgical exposure of the third ventricle floor obtained through the transcallosal-transchoroidal and transcallosal-transforniceal-transchoroidal approaches.

\section{Angular Exposure}

Angular exposure was obtained from the maximum viewing angles along the primary longitudinal and transverse axes of the third ventricle floor, obtained by transchoroidal and transforniceal-transchoroidal approaches. To format the third ventricle funnel, we defined 1 anterior and 1 posterior transverse axis and 1 anterior and 1 posterior longitudinal axis, yielding 4 values for each approach. The anterior transverse axis was defined as the greatest perpendicular distance between the fornix column and the 


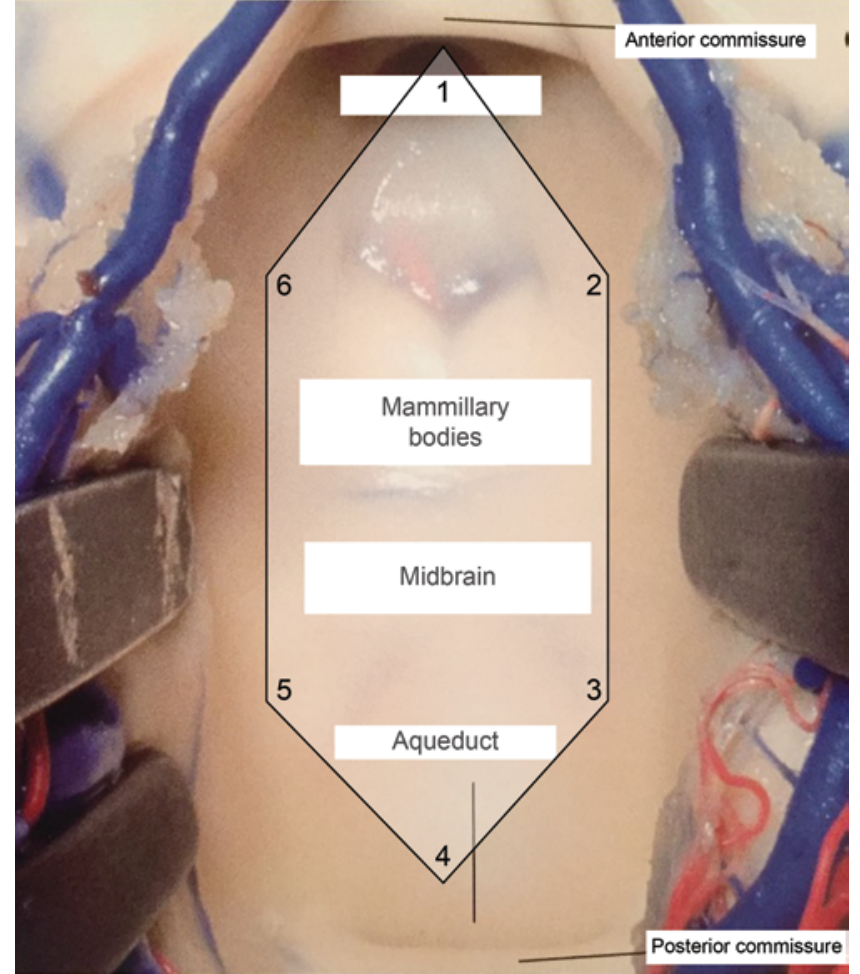

FIG. 4. Schematic representation of the microsurgical exposure area of the third ventricle floor. The 6 points corresponding to anatomical points of interest and delimiting a hexagonal area were as follows: the most anterior point exposed (1), point immediately most lateral and anterior to the floor of the right third ventricle (2), point immediately most lateral and posterior to the floor of the right third ventricle (3), cerebral aqueduct (4), point immediately most lateral and posterior to the floor of the left third ventricle (5), and point immediately most lateral and anterior to the floor of the left third ventricle (6). Adapted from Campero A, Ajler P, Emmerich J: Abordajes neuroquirúrgicos al cérebro y la base de crâneo, ed 1. Buenos Aires: Journal, 2013. Copyright Alvaro Campero. Published with permission. Figure is available in color online only.

anterior portion of the thalamus. The posterior transverse axis was defined as the greatest perpendicular distance between the fornix body and thalamus. The anterior and posterior longitudinal axes were defined as the line between the most distal point of the choroidal fissure after its dissection and the fornix column before and after its sectioning.

Two points were chosen in advance to evaluate angular exposure: the central tuber cinereum region for the anterior values and the cerebral aqueduct for the posterior values. These points were used to characterize the surgical targets most representative of the third ventricle floor. It was thus possible to evaluate and compare angular exposure between the 2 approaches.

Values were obtained using a neuronavigation system with customized software that systematically calculates angles on the different axes.

\section{Qualitative Analysis}

Measurements were obtained initially for the transcallosal-transchoroidal approach. We then sectioned the ipsilateral fornix column, which we called the transforniceal-

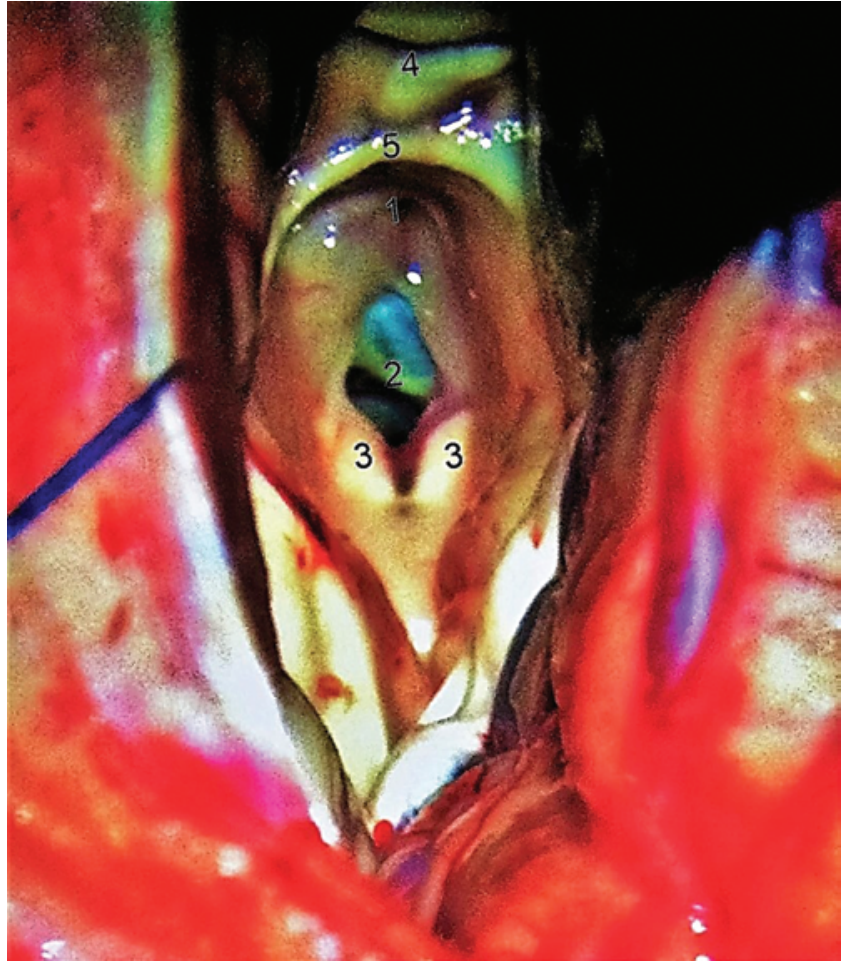

FIG. 5. Anatomical dissection of the specimen-microsurgical view of the anterior third ventricle region with posterior angulation of the microscope. 1 = infundibular recess of the pituitary gland; 2 = exposure of the interpeduncular cistern after opening the tuber cinereum; $3=$ mammillary bodies; 4 = right fornix column after section; 5 = anterior commissure. Figure is available in color online only.

transchoroidal approach. Using the anterior and posterior maximum angulation of the microscope, we defined the posterior and anterior exposure limits, respectively (Fig. 5). This methodology allowed us to visually compare the surgical exposure offered by the 2 approaches.

\section{Statistical Analysis}

Analysis of the results consisted of calculations of the following quantitative variables: mean, median, standard deviation, and amplitude ranges (minimum and maximum values). For inferential evaluation, the nonparametric Wilcoxon test was performed because the values did not have a normal distribution. The level of statistical significance adopted was $\mathrm{p}<0.05$.

\section{Results \\ Morphometry and Working Distance}

The mean thickness of the corpus callosum trunk was $10 \pm 1 \mathrm{~mm}$. The lowest value obtained was $8.5 \mathrm{~mm}$, and the highest was $12 \mathrm{~mm}$. The mean thickness of the right frontal lobe parenchyma was $35 \pm 3 \mathrm{~mm}$; the lowest value obtained was $30 \mathrm{~mm}$, and the highest was $41 \mathrm{~mm}$. The mean longitudinal diameter of the interventricular foramen was $4.6 \pm 0.4 \mathrm{~mm}$ (Table 1$)$.

\section{Surgical Exposure Area}

The mean value of the exposed area of the third ventri- 
TABLE 1. Values of morphometric evaluation

\begin{tabular}{lccccc}
\hline \multicolumn{1}{c}{ Variable } & Mean & SD & Median & Min & Max \\
\hline Corpus callosum thickness & 10 & 1 & 10 & 8.5 & 12 \\
\hline Parenchyma thickness, RFL & 35 & 3 & 34 & 30 & 41 \\
\hline IF diameter & 4.6 & 0.4 & 4.8 & 4.0 & 5.1 \\
\hline
\end{tabular}

IF = interventricular foramen; $\mathrm{RFL}=$ right frontal lobe.

Values are presented in millimeters.

cle floor for the transcallosal-transchoroidal approach was $80 \mathrm{~mm}^{2}$. After unilateral section of the fornix column, this value was $101 \mathrm{~mm}^{2}$, with a mean increase of $21 \mathrm{~mm}^{2}(\mathrm{p}<$ 0.05) (Fig. 6 and Table 2).

The mean value of the working area of the transcallosal-transchoroidal approach was $121 \mathrm{~mm}^{2}$ and that of the transcallosal-transforniceal-transchoroidal approach was $150 \mathrm{~mm}^{2}$. We therefore observed a mean increase of 29 $\mathrm{mm}^{2}(\mathrm{p}<0.05)$ in working area after sectioning the fornix column (Fig. 7 and Table 2).

\section{Angular Exposure}

No significant difference between the 2 approaches was found in the transversal anterior and posterior angular exposure. Sectioning of the fornix column therefore did not lead to an increase in transversal angular exposure of the 2 studied approaches.

In the anterior longitudinal angular exposure, the mean increase was $7^{\circ}$ after sectioning the fornix column, and the mean increase in posterior longitudinal exposure was $7^{\circ}(\mathrm{p}<0.05)$. Table 3 lists all of the values obtained.

\section{Qualitative Analysis of Surgical Exposure Area}

Using the maximum posterior and anterior angles of the microscope, the transchoroidal approach allowed for viewing of the posterior half of the tuber cinereum, mammillary bodies, midbrain surface (posterior perforated substance, medial parts of the bases of the cerebral pe-

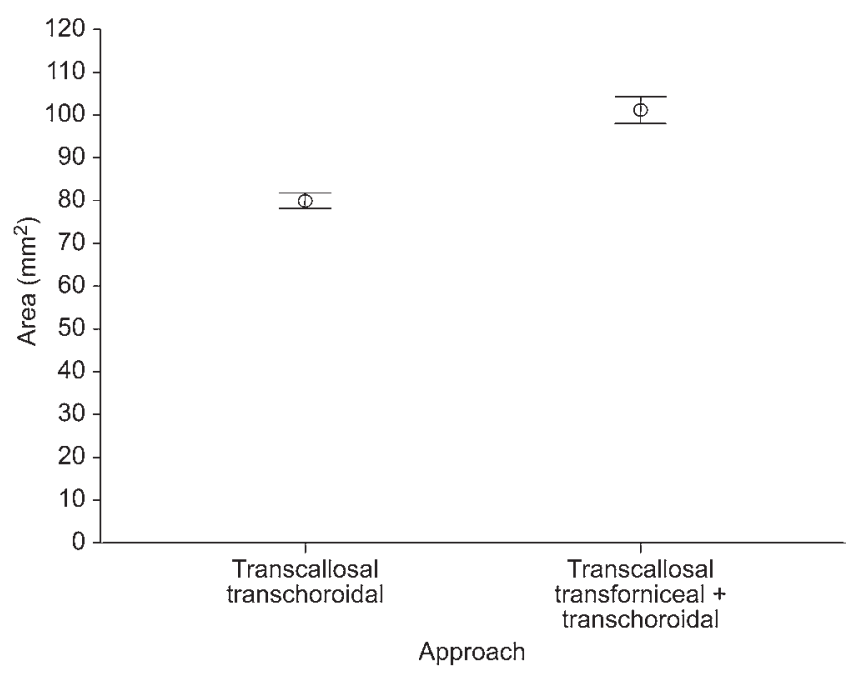

FIG. 6. Chart comparing the exposure area of the third ventricle floor between the 2 approaches. The bars correspond to \pm 2 SD.
TABLE 2. Exposure area of the third ventricle floor and working area of the 2 approaches

\begin{tabular}{lrrrrr}
\hline \multicolumn{1}{c}{ Variable } & Mean & SD & Median & Min & Max \\
\hline TC area & 80 & 5 & 80 & 71 & 85 \\
\hline TF + TC area & 101 & 9 & 98 & 91 & 115 \\
\hline TC working area & 121 & 8 & 120 & 112 & 137 \\
\hline TF + TC working area & 150 & 11 & 145 & 141 & 168 \\
\hline
\end{tabular}

$\mathrm{TC}=$ transchoroidal; $\mathrm{TF}+\mathrm{TC}=$ transforniceal-transchoroidal.

Values are presented in millimeters squared.

duncle and tegmentum), cerebral aqueduct, and posterior commissure.

Using the maximum anterior and posterior angulation, the transforniceal-transchoroidal approach allowed for the exposure of the pituitary infundibulum recess, the entire length of the tuber cinereum, mammillary bodies, mesencephalic surface (posterior perforated substance, medial portions of the cerebral peduncle and tegmentum bases), cerebral aqueduct, and posterior commissure (Fig. 8).

In both approaches, it was not possible to expose the posterior superior region of the third ventricle, due to the limited opening of the choroidal fissure. The transforniceal-transchoroidal approach did not allow for exposure of the anterior-upper region of the third ventricle, due to the obstructed view caused by the anterior commissure (Table 4).

\section{Discussion}

\section{Morphometric Analysis of Relevant Structures}

Classically, transcallosal access is the route of choice for performing the transchoroidal approach to the third ventricle and for operating on ventricular lesions located in the midline. ${ }^{2,40}$ However, in certain situations, such as in the presence of severe hydrocephalus with reduced thickness of the frontal lobe parenchyma or in cases of cerebral atrophy with a thickness less than $30 \mathrm{~mm}$, the transcorti-

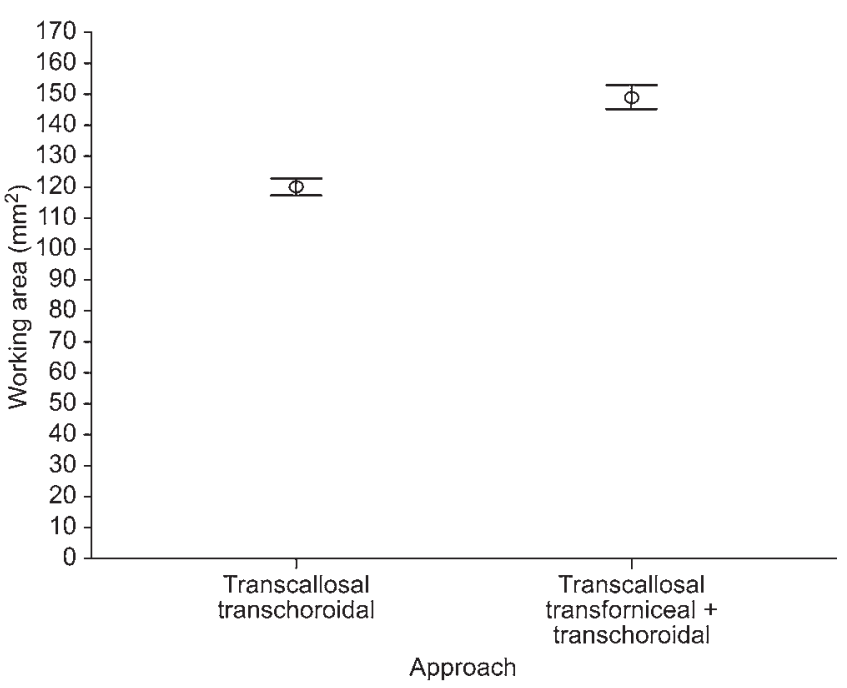

FIG. 7. Chart comparing the working area between the 2 approaches. The bars correspond to \pm 2 SD. 
TABLE 3. Comparison of angular exposures

\begin{tabular}{lccccc}
\hline \multicolumn{1}{c}{ Variable } & Mean & SD & Median & Min & Max \\
\hline ALAE TC $^{*}$ & 64 & 6 & 62 & 58 & 74 \\
\hline ALAE TF + TC & 71 & 7 & 71 & 60 & 78 \\
\hline ATAE TC & 38 & 6 & 35 & 30 & 46 \\
\hline ATAE TF + TC & 39 & 7 & 40 & 30 & 48 \\
\hline PLAE TC & 55 & 5 & 53 & 51 & 66 \\
\hline PLAE TF + TC & 62 & 6 & 62 & 54 & 74 \\
\hline PTAE TC & 28 & 3 & 29 & 24 & 32 \\
\hline PTAE TF + TC & 29 & 4 & 29 & 20 & 35 \\
\hline
\end{tabular}

$\mathrm{ALAE}=$ anterior longitudinal angular exposure; $\mathrm{ATAE}=$ anterior transversal angular exposure; PLAE = posterior longitudinal angular exposure; PTAE = posterior transversal angular exposure.

Values are presented in degrees.

* Values relating to anterior and posterior longitudinal exposure.

cal route can provide a greater exposed area of the lateral ventricle. ${ }^{29}$ According to the study by Nagasawa et al., the transcallosal route is superior to the transcortical, not only in the absence of ventricular dilation but also in situations of moderate hydrocephalus with parenchymal thickness between 30 and $40 \mathrm{~mm} .{ }^{29}$ Winkler et al. found a mean frontal lobe thickness of approximately $37.7 \mathrm{~mm}$ using the same parameters as the present study. ${ }^{41}$ In our study, we encountered a mean frontal lobe thickness of $35 \mathrm{~mm}$, and in all of the cadavers, the thickness was greater than $30 \mathrm{~mm}$. We therefore believe that in the present study, the choice

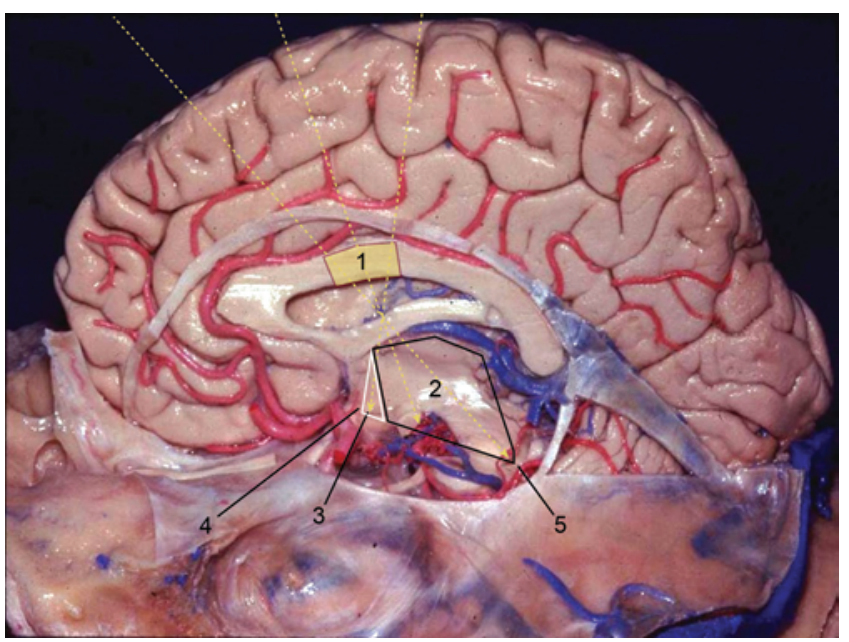

FIG. 8. Sagittal section of the brain fixed with formaldehyde-graphic representation, not to scale, of the microsurgical exposure of the 2 approaches. The black line marks the area and structures of the third ventricle exposed by the transchoroidal approach; the white line shows the gain in exposure obtained by sectioning the fornix column in the transforniceal-transchoroidal approach; and the dashed yellow line represents the anterior, posterior, and intermediate viewing angles of the microscope. 1 = callosotomy; 2 = microsurgical exposure area of the transchoroidal approach; 3 = triangular area corresponding to a gain of microsurgical exposure with sectioning of the ipsilateral column of the fornix; 4 = infundibular recess of the pituitary gland; 5 = cerebral aqueduct. Source: Modified with permission from an image provided by Drs. Hung Tzu Wen and Albert L. Rhoton. Figure is available in color online only.
TABLE 4. Representation of the presence or absence of exposure of the anatomical structures of the third ventricle by the respective approaches

\begin{tabular}{lcc}
\hline & \multicolumn{2}{c}{ View by Approach } \\
\cline { 2 - 3 } \multicolumn{1}{c}{ Structure } & & Transforniceal- \\
& Transchoroidal & Transchoroidal \\
\hline Supraoptic recess & - & - \\
\hline Infundibular recess & - & + \\
\hline Anterior half of the tuber cinereum & - & + \\
\hline Posterior half of the tuber cinereum & + & + \\
\hline Mammillary bodies & + & + \\
\hline Midbrain surface & + & + \\
\hline Cerebral aqueduct & + & + \\
\hline Posterior commissure & + & + \\
\hline
\end{tabular}

- = absence; + = presence.

of the transcallosal approach was appropriate and allowed for better exposure of the ventricular structures of interest.

The corpus callosum is the largest commissure of the brain and consists of more than 200 million fibers. It connects the 2 cerebral hemispheres, forming the ceiling of the lateral ventricles and the floor of the longitudinal brain fissure. ${ }^{31,32}$ The corpus callosum is divided into the rostrum, genu, trunk, and splenium. ${ }^{32}$ The thickness of the corpus callosum subregions differs with regard to ethnicity, sex, and the presence of diseases such as schizophrenia, Alzheimer disease, autism, and depression. ${ }^{19}$ The rostrum has a thickness of 2-3 mm; the genu, approximately 13 $\mathrm{mm}$; the middle portion of the trunk, approximately $6-12$ $\mathrm{mm}$; and the splenius, between 15 and $18 \mathrm{~mm} .{ }^{29,32}$

There is evidence that a longitudinal callosotomy of up to $2 \mathrm{~cm}$ in the proximal region of the corpus callosum trunk does not cause significant neuropsychological changes, making the anterior transcallosal route a safe alternative for accessing ventricular lesions. ${ }^{2,22,38,42}$ In the present study, we found a mean corpus callosum trunk thickness of approximately $10 \mathrm{~mm}$, which was similar to that found in other studies. ${ }^{19,31}$ Morphometric studies of individuals without neurological disease can most likely establish normal values of the corpus callosum subregions in a population to identify the presence and progression of a particular disease and to assist in the surgical planning of intraventricular lesions and some types of epilepsy.

The ventricular foramen was described by Scot Alexander Monro II in 1783 and consists of an elliptical space between the fornix column anteriorly and the anterior pole of the thalamus posteriorly. ${ }^{37}$ In studies by Fujii et al. and by Lang, the approximate longitudinal diameter was 5 $\mathrm{mm} \cdot{ }^{13,23,24}$ In the present study, we found a mean value of $4.6 \mathrm{~mm}$ in patients with no anatomical abnormalities or ventricular lesions. Yaşargil et al. reported that an expansion of the foramen of approximately 5-15 $\mathrm{mm}$ allows for so-called piecemeal-type resection of tumors located in the anterior two-thirds without causing inadvertent damage to regional structures ${ }^{44}$ For this reason, the individualized preoperative evaluation of the shape and size of the interventricular foramen can be helpful when choosing the most appropriate approach for each lesion. 


\section{Comparative Anatomical Analysis of Approaches}

The working area, or surgical freedom, is defined as a window in which the safe handling of surgical instruments is possible, and this is usually the prevalent limitation to target area exposure..$^{12}$ In the transchoroidal approach, the space obtained after opening the choroidal fissure is considered the working area and consists of the third ventricle ceiling. In the present study, we found a mean working area of $121 \mathrm{~mm}^{2}$ for the transchoroidal approach and 150 $\mathrm{mm}^{2}$ for the transforniceal-transchoroidal approach. Sectioning of the fornix column therefore led to an increase in working area of approximately $30 \mathrm{~mm}^{2}$. Despite the fact that the working area is often overlooked in anatomical studies, increasing the working area affords the surgeon larger viewing angles, better options when working with multidirectional perspectives, and greater comfort when performing the surgical procedure..$^{2}$

In the classic book, Surgery of the Third Ventricle, after an extensive literature review of the functional aspects and relevance of sectioning the fornix in the transforaminal approach to the third ventricle, Ehni and Ehni pose an important query: "A better question may be, does section of the fornix provide better exposure?" Answering this question has been one of the objectives of the present study.

The microsurgical exposure area corresponds to the anatomical area that allows for microscopic viewing and manipulation in the dissection of regional anatomical structures and resection of intracranial lesions. ${ }^{10,12}$ Based on this concept, the larger the area is, the greater the ability to dissect and expose the structures of the third ventricle, which promotes greater surgical safety. Ipsilateral sectioning of the fornix column quantitatively and qualitatively increased the exposure of the anterior third ventricle region structures. The transchoroidal approach provided a mean exposure area of $80 \mathrm{~mm}^{2}$. After sectioning the fornix column, the mean exposure area obtained was $101 \mathrm{~mm}^{2}$. These values objectively answer the main question formulated by Ehni and Ehni. ${ }^{9}$ From an anatomical view point, the values validate the use of this procedure in certain circumstances where an increase in microsurgical exposure of the anterior third ventricle region is needed..$^{13}$ Considering the great technical difficulty involved in operating on third ventricle lesions, increased exposure area decreases the risk of inadvertent manipulation of adjacent eloquent structures, with a consequent reduction in morbidity and an increased possibility of total resection of the lesion.

Qualitative evaluation revealed that the transfornicealtranschoroidal approach enabled the exposure of the anteroinferior, middle, and posteroinferior regions of the third ventricle. Exposure of the anterosuperior region, composed of the supraoptic recess and terminal blade, is limited by the anterior commissure and the impossibility of progress because the distal opening of the choroid fissure did not allow exposure of the posterosuperior region of the third ventricle. In accordance with Ulm et al. ${ }^{38}$ we observed that the transchoroidal approach is inadequate for accessing lesions in the anterior region or zone, because the fornix column limits exposure of that region. We therefore suggest its use, preferentially, for the middle and posteroinferior region of the third ventricle. The transfor- niceal-transchoroidal approach was proven to be versatile by allowing exposure of all 3 regions of the third ventricle. However, we believe that the anterosuperior and posterosuperior regions are better viewed by the translaminarterminal and transrecess-suprapineal approaches, respectively. Comparative anatomical studies would be useful to test this hypothesis.

Evaluating the effect of unilateral sectioning of the fornix column on cognition was not the aim of the present investigation. Several studies have demonstrated the safety of the technique and its insignificant cognitive repercussions. $^{2-4,7,8,15,21,22,28,30}$

Gaffan and Gaffan performed an extensive review of this topic and concluded that bilateral sectioning of the fornix causes memory deficits; however, there is no consistent evidence that unilateral sectioning of the fornix can affect memory. ${ }^{14}$ McMackin et al. evaluated the neuropsychological aspects of 6 patients undergoing surgery for removal of a third ventricle colloid cyst. They observed that bilateral lesions of the fornix in 5 patients caused a consequent deficit in short-term memory. However, the patient with a unilateral lesion of the right fornix recovered without any change to his memory and made a full return to work activities. ${ }^{27}$

Aggleton et al. showed that only bilateral interruption of the fornix, evidenced by brain MRI, proved to be a predictor of decreased memory performance in 12 patients who had surgical treatment of colloid cysts in the third ventricle. ${ }^{1}$ After evaluating 38 patients who underwent removal of colloid cysts from the third ventricle, Tsivilis et al. ${ }^{36}$ concluded that the reduction in the volumes of the left fornix and left mammillary body shown on MRI were consistently related to memory impairment.

We believe that further studies, with good methodological quality, must be conducted to better understand the interaction between the fornix column and the other brain structures involved in memory. Currently, there are no studies objectively showing that unilateral, surgically induced injury to the right fornix (nondominant hemisphere) in humans has a significant impact on memory. Based on this information, its use may be suggested in selected cases after proper neuropsychological evaluation, in situations where greater surgical exposure of the anterior third ventricle region is required.

Angular exposure is increased with bone removal, cerebral retraction, or the sectioning of structures. Greater angulation allows the surgeon to work in various directions, which promotes greater safety during microsurgical dissection of neurovascular structures, with a consequent reduction in the risk of unnecessary manipulation of the surrounding nerve tissue. ${ }^{9-11}$ Angular exposure can also be understood as the surgeon's range of executable movements. ${ }^{12}$ Longitudinal and transverse exposure was evaluated using the tuber cinereum in the anterior region and the cerebral aqueduct in the posterior region. Thus, in each approach, 2 values were obtained for longitudinal exposure and 2 for transversal exposure.

The evaluation of 1 anterior and 1 posterior parameter was justified by the funnel format of the third ventricle. There was no difference in transverse angular exposure between the 2 approaches; however, there were mean in- 
creases of approximately $7^{\circ}$ in longitudinal anterior angular exposure and approximately $7^{\circ}$ in longitudinal posterior angular exposure. This result indicates that the transforniceal-transchoroidal approach allows for more angulation in the longitudinal plane, which may result in safer resection of extensive lesions occupying the anterior, middle, and posterior regions of the third ventricle.

To our knowledge, this study is the first to use fresh cadavers to systematically compare 2 approaches to the third ventricle using a neuronavigation system. In addition to performing a morphometric study of the relevant structures, we quantitatively evaluated microsurgical exposure using 6 parameters and compared the data obtained for the transchoroidal and transforniceal-transchoroidal approaches. In addition, a qualitative evaluation of the approaches was performed, objectively exposing their limitations and optimal applicability in neurosurgical practice.

This method provided a comparative anatomical study of 2 approaches in a single situation, that is, in cadavers without anatomical changes or distortions of the third ventricle. However, in neurosurgical practice, what motivates access to this area are precisely the lesions that usually occupy and alter ventricular morphology. Therefore, it is essential to perform a thorough and individualized analysis of each case. Other limitations of this study relate to the consistency of the brain in the cadaver and the absence of blood and CSF flow. These factors may affect the technical difficulty of implementing these surgical approaches.

\section{Conclusions}

The transcallosal-transforniceal-transchoroidal approach provides a greater microsurgical exposure area, greater working area, and greater angular exposure area in the longitudinal plane than the transcallosal-transchoroidal approach. There was no difference in angular exposure on the transverse plane between the 2 approaches. Qualitative evaluation showed that the transcallosal-transforniceal-transchoroidal approach allows for exposure of the infundibular recess and front half of the tuber cinereumstructures that are not exposed by a transcallosal-transchoroidal approach. Morphometric analysis established mean values for the following structures: frontal lobe parenchyma thickness $(35 \mathrm{~mm})$, corpus callosum trunk thickness $(10 \mathrm{~mm})$, and longitudinal diameter of the interventricular foramen $(4.6 \mathrm{~mm})$ in the studied population.

\section{References}

1. Aggleton JP, McMackin D, Carpenter K, Hornak J, Kapur N, Halpin S, et al: Differential cognitive effects of colloid cysts in the third ventricle that spare or compromise the fornix. Brain 123:800-815, 2000

2. Bellotti C, Pappadà G, Sani R, Oliveri G, Stangalino C: The transcallosal approach for lesions affecting the lateral and third ventricles. Surgical considerations and results in a series of 42 cases. Acta Neurochir (Wien) 111:103-107, 1991

3. Bengochea FG, De La Torre O, Esquivel O, Vieta R, Fernan$\operatorname{dez} C$ : The section of the fornix in the surgical treatment of certain epilepsies; a preliminary report. Trans Am Neurol Assoc 13 (79th Meeting):176-178, 1954

4. Cairns H, Mosberg WH Jr: Colloid cyst of the third ventricle. Surg Gynecol Obstet 92:545-570, 1951
5. Campero A, Ajler P, Emmerich J: Abordajes neuroquirúrgicos al cérebro y la base de crâneo, ed 1. Buenos Aires: Journal, 2013

6. Chang EF, Gabriel RA, Potts MB, Berger MS, Lawton MT: Supratentorial cavernous malformations in eloquent and deep locations: surgical approaches and outcomes. Clinical article. J Neurosurg 114:814-827, 2011

7. Dandy WE: Benign Tumors in the Third Ventricle of the Brain: Diagnosis and Treatment. Springfield, IL: C.C. Thomas, 1933

8. Desai KI, Nadkarni TD, Muzumdar DP, Goel AH: Surgical management of colloid cyst of the third ventricle-a study of 105 cases. Surg Neurol 57:295-304, 2002

9. Ehni G, Ehni B: Considerations in transforaminal entry, in Apuzzo MLJ (ed): Surgery of the Third Ventricle, ed 2. Baltimore: Williams \& Wilkins, 1998, pp 326-353

10. Figueiredo EG, Deshmukh P, Nakaji P, Crusius MU, Crawford N, Spetzler RF, et al: The minipterional craniotomy: technical description and anatomic assessment. Neurosurgery 61 (5 Suppl 2):256-265, 2007

11. Figueiredo EG, Deshmukh P, Zabramski JM, Preul MC, Crawford NR, Spetzler RF: The pterional-transsylvian approach: an analytical study. Neurosurgery 62 (6 Suppl 3):1361-1367, 2008

12. Figueiredo EG, Zabramski JM, Deshmukh P, Crawford NR, Spetzler RF, Preul MC: Comparative analysis of anterior petrosectomy and transcavernous approaches to retrosellar and upper clival basilar artery aneurysms. Neurosurgery 58 (1 Suppl):ONS13-ONS21, 2006

13. Fujii K, Lenkey C, Rhoton AL Jr: Microsurgical anatomy of the choroidal arteries: lateral and third ventricles. J Neurosurg 52:165-188, 1980

14. Gaffan D, Gaffan EA: Amnesia in man following transection of the fornix. A review. Brain 114:2611-2618, 1991

15. Garcia-Bengochea F, Friedman WA: Persistent memory loss following section of the anterior fornix in humans. A historical review. Surg Neurol 27:361-364, 1987

16. Garretson HD: Commentary A - memory in man: a neurosurgeons perspective, in Apuzzo MLJ (ed): Surgery of the Third Ventricle, ed 2. Baltimore: Williams \& Wilkins, 1998, pp 209-212

17. Hernesniemi J, Leivo S: Management outcome in third ventricular colloid cysts in a defined population: a series of 40 patients treated mainly by transcallosal microsurgery. Surg Neurol 45:2-14, 1996

18. Kandel E, Kupfermann I, Iversen S: Learning and memory, in Kandel E, Schwartz J, Jessell T (eds): Principles of Neural Science, ed 4. New York: McGraw-Hill, 2000

19. Karakaş P, Koç Z, Koç F, Gülhal Bozkır M: Morphometric MRI evaluation of corpus callosum and ventricles in normal adults. Neurol Res 33:1044-1049, 2011

20. Konovalov AN, Gorelyshev SK: Surgical treatment of anterior third ventricle tumours. Acta Neurochir (Wien) 118:33-39, 1992

21. Konovalov N: Technique and strategies of direct surgical management of craniopharyngioma, in Apuzzo MLJ (ed): Surgery of the Third Ventricle. Baltimore: Williams \& Wilkins, 1998, pp 542-553

22. Koos WT, Perneczky A, Horaczek A: Problems of surgical technique for the treatment of supratentorial midline tumors in children. Acta Neurochir Suppl 35:31-41, 1985

23. Lang J: Anatomy of the midline. Acta Neurochir Suppl 35:6-22, 1985

24. Lang J: Topographic anatomy of preformed intracranial spaces. Acta Neurochir Suppl 54:1-10, 1992

25. Little JR, MacCarty CS: Colloid cysts of the third ventricle. J Neurosurg 40:230-235, 1974

26. Mazarakis NK, Summers F, Murray AD, Waiter GD, Fouyas IP: Partial recovery from amnesia following bilateral surgical 
fornix transection is correlated with cortical plasticity. $\mathbf{B r} \mathbf{J}$ Neurosurg 25:658-661, 2011

27. McMackin D, Cockburn J, Anslow P, Gaffan D: Correlation of fornix damage with memory impairment in six cases of colloid cyst removal. Acta Neurochir (Wien) 135:12-18, 1995

28. Morita A, Kelly PJ: Resection of intraventricular tumors via a computer-assisted volumetric stereotactic approach. Neurosurgery 32:920-927, 1993

29. Nagasawa S, Miyake H, Ohta T: [Transcallosal and transcortical approaches for tumors at the anterior part of the lateral ventricle: relations between visualized and ventricular size.] No Shinkei Geka 25:321-327, 1997 (Jpn)

30. Patel P, Cohen-Gadol AA, Boop F, Klimo P Jr: Technical strategies for the transcallosal transforaminal approach to third ventricle tumors: expanding the operative corridor. J Neurosurg Pediatr 14:365-371, 2014

31. Peltier J, Verclytte S, Delmaire C, Deramond H, Pruvo JP, Le Gars D, et al: Microsurgical anatomy of the ventral callosal radiations: new destination, correlations with diffusion tensor imaging fiber-tracking, and clinical relevance. J Neurosurg 112:512-519, 2010

32. Rhoton AL Jr: The cerebrum. Anatomy. Neurosurgery 61 (1 Suppl):37-119, 2007

33. Sekhar LN, Fessler RG: Atlas of Neurosurgical Techniques: Brain. New York: Thieme, 2006

34. Shucart W: Anterior transcallosal and transcortical approaches, in Apuzzo MLJ (ed): Surgery of the Third Ventricle, ed 2. Baltimore: Williams \& Wilkins, 1998, pp 303-325

35. Tomasello F, Cardali S, Angileri FF, Conti A: Transcallosal approach to third ventricle tumors: how I do it. Acta Neurochir (Wien) 155:1031-1034, 2013

36. Tsivilis D, Vann SD, Denby C, Roberts N, Mayes AR, Montaldi D, et al: A disproportionate role for the fornix and mammillary bodies in recall versus recognition memory. Nat Neurosci 11:834-842, 2008

37. Tubbs RS, Oakes P, Maran IS, Salib C, Loukas M: The foramen of Monro: a review of its anatomy, history, pathology, and surgery. Childs Nerv Syst 30:1645-1649, 2014

38. Ulm AJ, Russo A, Albanese E, Tanriover N, Martins C, Mericle RM, et al: Limitations of the transcallosal transchoroidal approach to the third ventricle. J Neurosurg 111:600-609, 2009
39. Wen H: Demonstração anatômica da abordagem transcorioidea à porção anterior do terceiro ventrículo [thesis]. São Paulo: Faculdade de Medicina, Universidade de São Paulo, 2002

40. Wen HT, Rhoton AL Jr, de Oliveira E: Transchoroidal approach to the third ventricle: an anatomic study of the choroidal fissure and its clinical application. Neurosurgery 42:1205-1219, 1998

41. Winkler PA, Weis S, Büttner A, Raabe A, Amiridze N, Reulen HJ: The transcallosal interforniceal approach to the third ventricle: anatomic and microsurgical aspects. Neurosurgery 40:973-982, 1997

42. Woiciechowsky C, Vogel S, Lehmann R, Staudt J: Transcallosal removal of lesions affecting the third ventricle: an anatomic and clinical study. Neurosurgery 36:117-123, 1995

43. Woolsey RM, Nelson JS: Asymptomatic destruction of the fornix in man. Arch Neurol 32:566-568, 1975

44. Yaşargil MG: Microneurosurgery. Stuttgart: Thieme, 1996

\section{Disclosures}

The authors report no conflict of interest concerning the materials or methods used in this study or the findings specified in this paper.

\section{Author Contributions}

Conception and design: Vitorino Araujo, Figueiredo. Acquisition of data: Vitorino Araujo, Otoch. Analysis and interpretation of data: Vitorino Araujo, Veiga, Wen, Figueiredo. Drafting the article: Vitorino Araujo, Rhoton, Figueiredo. Critically revising the article: Vitorino Araujo, Veiga, Wen, de Andrade, Teixeira, Preul, Spetzler, Figueiredo. Reviewed submitted version of manuscript: Vitorino Araujo, Veiga, de Andrade, Teixeira, Rhoton, Preul, Spetzler, Figueiredo. Approved the final version of the manuscript on behalf of all authors: Vitorino Araujo. Statistical analysis: Vitorino Araujo. Administrative/technical/material support: Vitorino Araujo, Veiga, Wen, Teixeira, Otoch, Figueiredo. Study supervision: Vitorino Araujo, Figueiredo.

\section{Correspondence}

João Luiz Vitorino Araujo, Cesário Mota Júnior Street, 112 Vila Buarque, São Paulo 01221-020, Brazil. email: vitorinomed@ yahoo.com.br. 